\title{
Free-electron qubits and maximum-contrast attosecond pulses via temporal Talbot revivals
}

\author{
M. V. Tsarev ๑, A. Ryabov ๑, and P. Baum $\odot^{*}$ \\ Universität Konstanz, Universitätsstraße 10, 78464 Konstanz, Germany
}

(Received 15 April 2021; accepted 14 September 2021; published 14 October 2021)

\begin{abstract}
We use laser light and a transmission electron microscope to modulate a free-electron beam into high-contrast electron pulses and free-electron qubits by using temporal Talbot revivals. At large enough propagation distances, the discrete energy sidebands from a laser modulation acquire special phases and group delays that optimize or cancel their time-domain interference, producing a revival or alternatively a pulse train at close to $100 \%$ modulation depth. A sequence of two laser interactions at an optimized propagation distance allows us to coherently control adjacent energy sidebands in amplitude and phase in the way of a qubit. The use of continuous-wave laser light provides these modulations at almost the full brightness of the beam source. Free electrons under large-distance laser control are therefore a promising tool for ultrafast material characterizations or investigations of free-electron quantum mechanics.
\end{abstract}

DOI: 10.1103/PhysRevResearch.3.043033

\section{INTRODUCTION}

The electron microscope is one of the most versatile instruments for investigating the atomic structure of complex materials, but it is also useful for understanding the quantum mechanics of the free electron and its strong interactions with coherent and incoherent light [1-6]. For characterizations of ultrafast material dynamics, electrons can be modulated in time by the cycles of laser light [7-10], providing femtosecond and attosecond time resolution for applications in photon-induced electron microscopy [11,12], ultrafast electron diffraction [9], or waveform electron microscopy [13,14]. At these ultimately fast time scales, the structure of pulsed beam electrons is dominated by their wave properties, evident from recent observations of discrete energy sidebands $[11,15,16]$, Rabi oscillations [17], quantum random walks $[8,18,19]$, and Hanbury-Brown-Twiss anticorrelations [20]. Consequently, ultrashort electron pulse formation is linked to quantum effects [17,21].

Here, we ask how attosecond imaging can reach optimum conditions for diffraction and microscopy experiments [9] and how a free electron as an elementary particle can eventually become a potential carrier of quantum information [22]. To this end, we combine a transmission electron microscope with two continuous-wave laser beams. Contrary to previous experiments, we apply a particularly large free-space propagation time that exceed $10^{5}$ times the laser cycle period [10]. We find a complex sequence of matter-wave transformations: Initially, the electron wave packet compresses into a series of ultra-

\footnotetext{
*peter.baum@uni-konstanz.de

Published by the American Physical Society under the terms of the Creative Commons Attribution 4.0 International license. Further distribution of this work must maintain attribution to the author(s) and the published article's title, journal citation, and DOI.
}

short electron pulses; next, these pulses lengthen to half the inverse modulation frequency at the benefit of zero temporal background; then the wave packet turns again into the same ultrashort pulses as before; and finally, the wave function experiences an almost full restoration to the original state after the initial laser modulation. This chain of transformations and revivals recurs multiple times upon further propagation [23] and is robust against decoherence or dispersion. Consequently, a second laser interaction at a properly chosen fractional revival time allows us to demonstrate experimental evidence for the production of a free-electron qubit.

\section{EXPERIMENTAL IDEA}

Figure 1(a) shows the idea of the experiment. An electron beam (blue) passes through a thin membrane (orange) illuminated by laser light (red). At proper angles and polarization [9], the electric fields of the optical cycles impose a periodic modulation of forward momentum. In the energy domain, the spectrum obtains a discrete set of sidebands whose separation is linked to the photon energy [11,15-17]. In the time domain, faster electrons gradually catch up with slower ones, and the electron beam transforms into a sequence of ultrashort pulses with femtosecond or attosecond durations [8,9], albeit with a temporal background from the decompressing electrons from adjacent modulation cycles $[8,9]$. Behind this temporal focus, the pulses overdisperse, and the temporal structure falls apart [9].

However, at an even longer propagation distance beyond this regime, the accelerated part of each pulse will catch up with the decelerated part of each preceding pulse $[23,24]$. We might therefore hope, guided by simulations [22,23,25], that quantum interferences can restore the original wave function [23], form attosecond electron pulses with optimized temporal contrast [25], or prepare special temporal structures for subsequent manipulations with multiple laser beams. A 

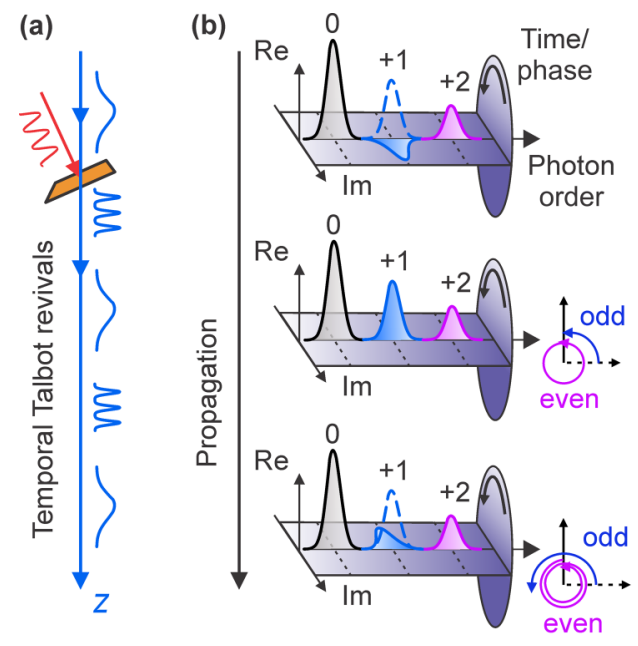

(c)

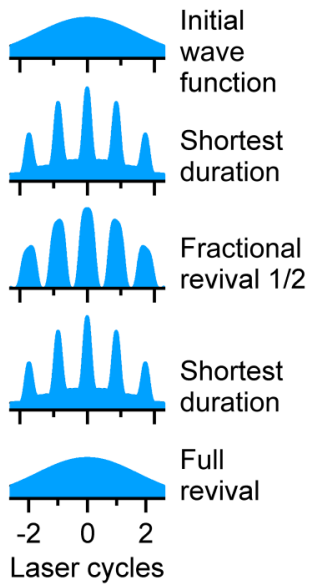

(d)

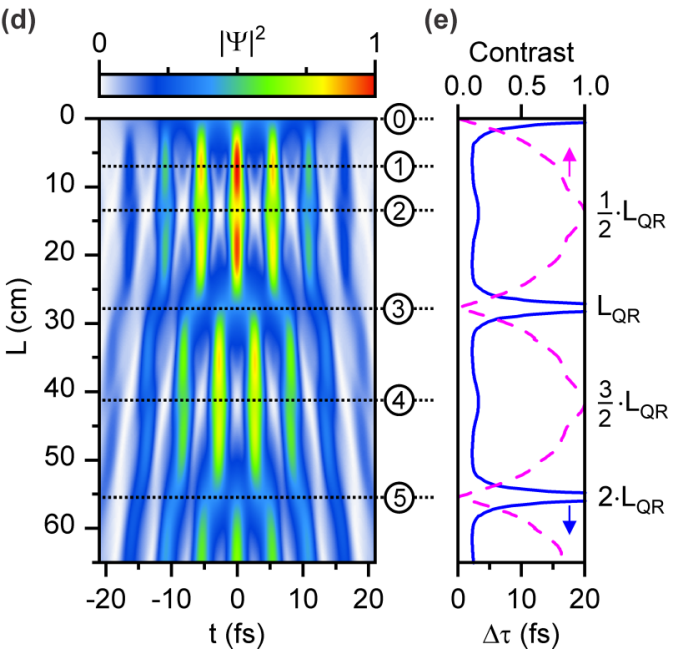

FIG. 1. Quantum revivals and sub-light-cycle electron microscopy at maximum contrast. (a) Concept for exploiting quantum revivals for generating attosecond electron pulses and qubits. A laser field (red) modulates an electron beam (blue) at a dielectric membrane (orange). Upon further propagation, the wave packet transforms into pulses and afterward restores back to the initial form, followed by further pulse compressions and revivals. (b) Evolution of the energy spectrum and sideband phases. Upper panel: Initial phases. Middle panel: Phases at the half-revival. Bottom panel: Phases at the full revival. (c) Wave packets and pulses in the time domain. A Gaussian envelope is added for depicting a limited temporal coherence. (d) Simulated quantum carpet $|\Psi|^{2}$ of an electron wave packet at a kinetic energy of $75 \mathrm{keV}$. $L$ is the propagation distance, and $t$ is the local time. (e) Electron pulse duration $\Delta \tau$ (solid) and temporal contrast (dashed) as a function of $L$. Pulse duration is defined as the full width at half maximum of the peak above the adjacent local minimum $[8,9]$.

free-electron qubit may be formed with laser modulations at special propagation distances [22].

\section{TEMPORAL TALBOT REVIVALS}

Before reporting ultrashort pulses or qubits, we consider the temporal dynamics of a laser-prepared electron wave packet after extended propagation times. In the spectral domain [see Fig. 1(b)], the free-electron wave function after laser modulation consists of a discrete set of spectral sidebands that are separated by the laser photon energy $h v$ [15-17]; $v$ is the laser frequency, and $h$ is Planck's constant. Every second sideband has a $90^{\circ}$ phase shift according to a Jacobi-Anger expansion [11,26]. Electrons have a rest mass and consequently a nonlinear energy-momentum relation. During free-space propagation, the sidebands therefore accumulate a series of additional phases that depend on the sideband order $n$. This dependency is quadratic because the laser-induced energy changes (a few electronvolts) are negligible with respect to the central energy of the electrons (tens of kiloelectronvolts). Application of a relativistically modified Schrödinger equation [27] is therefore appropriate, and we can find an analytical quantum-mechanical solution (see Appendix A).

The free-space rotation of the sideband phases will first lead to alignment [Fig. 1(b), middle panel] and then produce a phase-mirrored initial configuration (bottom panel). From our analytical theory, we derive a revival distance of $L_{\mathrm{QR}}=d^{2} / \lambda_{e}$, where $\lambda_{e}=h /\left(\gamma m_{e} v_{0}\right)$ is the de Broglie wavelength of the electron, $m_{e}$ is the mass of the electron, $\gamma$ is the Lorentz factor, $v_{0}$ is the electron velocity, $d=\beta \gamma \lambda$ is the effective modulation wavelength, $c$ is the speed of light, $\beta=v_{0} / c$, and $\lambda$ is the wavelength of the laser. At this $L_{\mathrm{QR}}$, the first sideband will have a phase equal to $\pi$, while the $n$th sideband accumu- lates a phase of $n^{2} \pi$ (lower panel). Consequently, the electron density as a function of time at $L_{\mathrm{QR}}$ will be indistinguishable from the initial one. A complete restoration including phases occurs at $L=2 L_{\mathrm{QR}}$. This phenomenon [23] is a time-domain analogue to the Talbot effect in optics [28,29], and we call the propagation time toward the first revival the temporal Talbot time $T_{\mathrm{QR}}=d^{2} /\left(\lambda_{e} v_{0}\right)$.

There will also be fractional revivals: If only some of the sidebands have phases equal to multiples of $2 \pi$ while other sidebands have arbitrary phases, only some of the spectral components will constructively restore the time-domain wave function, while the other sidebands will produce destructive interference. Figure 1(c) depicts such electron densities in the time domain. Particularly interesting is the case of the fractional revival of the order $\frac{1}{2}$ at $L=L_{\mathrm{QR}} / 2$, shown in the middle panel of Fig. 1(b). At this condition, the odd sidebands are brought to the same phase as the even sidebands, and in the time domain, there will be maximum destructive interference. As a result, we expect the formation of a train of attosecond electron pulses with $100 \%$ contrast [see Fig. 1(c), middle panel] and without the constant background current of $\sim 30 \%$ that has been ubiquitous in attosecond electron microscopy so far $[8,9]$.

Figure 1(d) shows a calculation of the quantum dynamics of a free-electron wave function at realistic beam parameters (Appendix B). We use an electron energy of $75 \mathrm{keV}$, a laser wavelength of $1.95 \mu \mathrm{m}$, a laser-electron coupling constant of $|g|=0.785$, and an initial Gaussian energy spread of 0.03 $\mathrm{eV}$ (root mean square) [30]. Indeed, we see in Fig. 1(d) the expected dynamics: first, the temporal focusing of the initial electron current density to a train of ultrashort pulses of minimum duration at the propagation distance (1); next, at $L=L_{\mathrm{QR}} / 2$, the formation of pulses with $100 \%$ contrast at the distance (2) followed at $L=L_{\mathrm{QR}}$ by a nearly full revival of the 
(a)

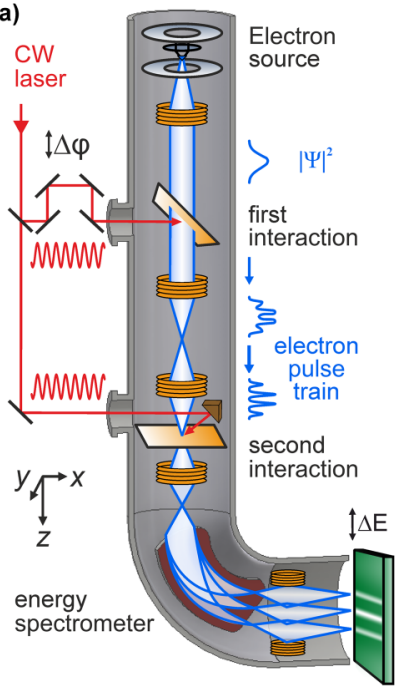

(b)

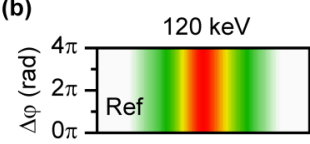

(c)

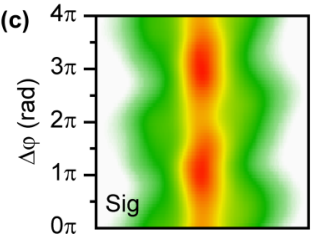

(d)

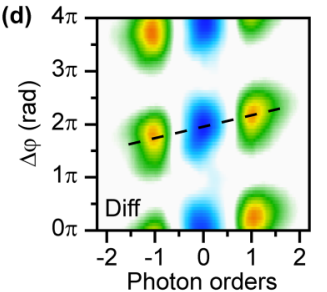

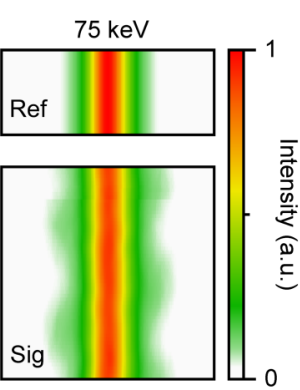

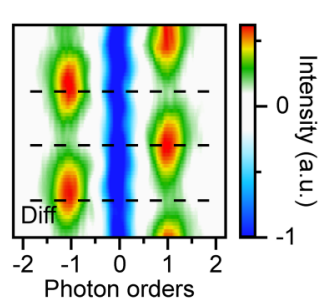

(e)
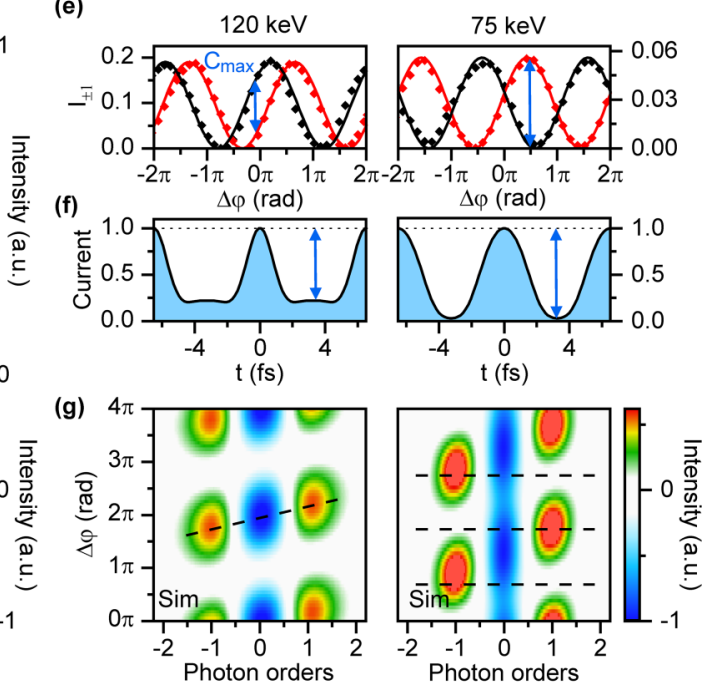

FIG. 2. Concept and experiment for attosecond pulse formation and free-electron quantum optics. (a) Schematic of the instrument. A continuous-wave (CW) laser (red) interacts with electron wave packets $\Psi$ (blue) in a transmission electron microscope at two interaction regions (orange), one for temporal compression and one for diagnostics or qubit formation. The optical phase difference $\Delta \varphi$ is controlled with a piezoelectric actuator. Electron energy spectra are dispersed with a magnetic spectrometer on a screen (green). (b)-(d) Measurement results for an electron energy of $120 \mathrm{keV}$ (left panels) and $75 \mathrm{keV}$ (right panels). (b) The laser-unaffected reference spectrum is independent of laser delay $\Delta \varphi$. (c) An electron spectrum after two interactions with laser light as a function of $\Delta \varphi$. (d) Difference between the signal spectrum from (c) and the reference spectrum from (b). The intensity is normalized to the decrease in the zero-loss peak. Dashed lines are indications of symmetry. The laser powers at the two membranes are $4 \mathrm{~W}$ for $120 \mathrm{keV}$ electrons and $3 \mathrm{~W}$ for $75 \mathrm{keV}$ electrons, corresponding to electric field strengths of 2 and $1.7 \mathrm{MV} / \mathrm{m}$, respectively. (e) Measurement and analysis of the \pm 1 photon-order sideband intensities $I_{ \pm 1}$ for $120 \mathrm{keV}$ (left panel) and $75 \mathrm{keV}$ (right panel). Intensities are normalized to the laser-unaffected zero-loss peak. (f) Electron currents for the conditions of short-pulse formation (left) and maximum contrast (right). The blue arrows show the pulse contrast $C_{\max }$ (peak above background). (g) Analytical predictions obtained by fitting probability density of Eq. (A16) to the experimental data of (d).

initial electron current at the distance (3). After this restoration, the entire process repeats, and the current density periodically compresses back to pulses (e.g., at distance (4)) and higher-order revivals (e.g., at distance (5)). The limited temporal coherence of the initial wave function $(\sim 10 \mathrm{fs})$ causes some overall dispersion effects that spread the wave packet and make higher-order revivals slightly nonideal. Figure 1(e) shows the evolution of the electron pulse duration $\Delta \tau$ and the degree of temporal contrast as a function of propagation distance. Shortly after the interaction, the shortest pulses are formed, and $\Delta \tau$ reaches its minimum. Afterward, $\Delta \tau$ reaches at the half-revival point $L_{\mathrm{QR}} / 2$ a local maximum that only slightly exceeds the minimal value. Next, the pulses shorten to minimum duration for a second time. The temporal contrast, defined here as the ratio of electron current within the pulses against the constant-current background, rises from zero to unity upon propagation from zero to $L_{\mathrm{QR}} / 2$ and then goes back to zero at the full revival, where the initial electron density is restored. Free electrons therefore exhibit very similar revival dynamics to isolated atoms and molecules [31-33], Bose-Einstein condensates [34-38], or spin systems [39].

\section{EVIDENCE FOR ULTRASHORT ELECTRON PULSES AT MAXIMUM CONTRAST}

Figure 2(a) illustrates the experimental setup. A Philips CM120 transmission electron microscope (gray) was upgraded with two electron-laser interactions. The first laserelectron interaction (compression) was located at the con- denser aperture (9 $\mathrm{cm}$ after the source), and the second interaction (attosecond diagnostics/qubit formation) was in the sample chamber. Both times, a continuous-wave laser (AdValue Photonics) at $\lambda=1.95 \mu \mathrm{m}$ interacted with the electron beam at a thin silicon membrane [10]. The interaction elements were 50-nm-thick ultraflat, highly doped silicon membranes with an aperture of $\sim 100 \times 200 \mu \mathrm{m}^{2}$ (UberFlat, Norcada). The electrons obtained a time-dependent momentum modulation and discrete energy sidebands at $h v=$ $\pm 0.64 \mathrm{eV}$. The electrons propagated for $\sim 12 \mathrm{~cm}$ of free space between the two interactions. Adjustment of the electron energy $E_{0}$ between 75 and $120 \mathrm{keV}$ allowed us to change the propagation time between the two interactions. In the end, the energy spectrum of the electrons was measured with a magnetic energy spectrometer (Gatan GIF 100) as a function of the laser phase delay $\Delta \varphi$, adjusted by a mirror on a piezoelectric actuator. The electron current was set to $\sim 10$ pA to minimize energy bandwidth and maximize temporal coherence. Electromagnetic laboratory noise was filtered out by drift tube chopping [10].

Figures 2(b)-2(d) show the measured energy spectrograms for two cases $E_{0}=120 \mathrm{keV}$ (left) and $E_{0}=75 \mathrm{keV}$ (right). According to the analytical predictions (see Appendix A), $120 \mathrm{keV}$ corresponds to generating electron pulses with shortest duration [10], whereas $75 \mathrm{keV}$ should roughly correspond to a half-revival distance between the two interactions. In both sets of data, we observe periodic side peaks at $\pm h v$, but their dynamics is different. For $120 \mathrm{keV}$, the positive and negative sideband peaks are found at nonsymmetric time delays, indi- 
(a)

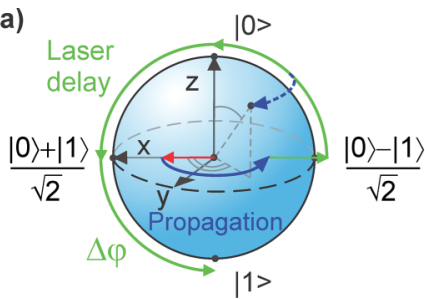

(b)

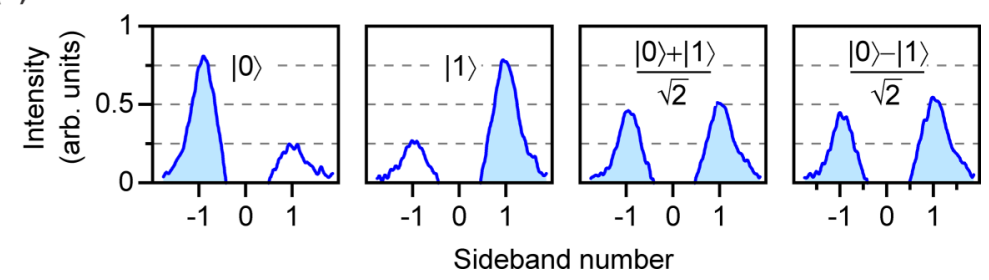

FIG. 3. Experimental evidence for realization of a free-electron qubit. (a) Bloch sphere. State $|0\rangle$ denotes an electron wave function in the -1 energy sideband, and state $|1\rangle$ denotes an electron wave function in the +1 sideband. In the experiment, the first laser-electron interaction (red) creates from the zero-loss peak a symmetric sideband pattern. Free propagation for half the temporal Talbot time (solid blue) rotates the phases and simultaneously prepares a time-controlled wave function. The second laser interaction (green) creates the qubit and simultaneously rotates it around the Bloch sphere in dependence of the optical laser phase delay $\Delta \varphi$. Additional free-space propagation or adjusting the timing of electron emission can rotate the qubit state around the $z$ axis (dashed blue). Note that the transient Bloch sphere with smaller radius (red arrow) simply indicates less electron probability in the sidebands and not a statistical incoherence. (b) Measured electron spectra for the four particular qubit states depicted in the Bloch sphere. Phases are discussed in the text. The zero-loss peak [constant with delay time; see Fig. 2(b)] is subtracted for clarity.

cated by the tilted dashed lines [Fig. 2(d), left panel]. Also, there are minima in the zero-loss peak that are periodic with the laser delay. Consequently, electron current redistributes in a complex way between all three energy features as a function of $\Delta \varphi$, and no peak is ever purely on or off. The origin of this observation is the finite propagation time that is neither negligible nor corresponds to a particular fractional revival.

In contrast, the data for $75 \mathrm{keV}$ [Fig. 2(d), right panel] reveal positive and negative sidebands that alternate almost precisely between purely positive and negative energy changes as a function of laser delay. The zero-loss peak shows no modulation, and its electron current is independent of the laser delay. The energy-gain peak $(+1)$ is anti-aligned with the loss peak $(-1)$, as indicated by the dashed lines. This emergence of symmetry occurs for an even longer free-space propagation time than for $120 \mathrm{keV}$, and the result therefore provides direct indication of wave function revivals in the time domain.

Figure 2(e) shows the fitted intensities of the \pm 1 sidebands and the zero-loss peak for each laser phase delay. For both electron energies, the sidebands exhibit sinusoidal dependencies on the delay, but there are differences in the positions of the minimum value and different phase shifts. For $120 \mathrm{keV}$, the phase shift between the two sinusoidals is $\sim 120^{\circ}$, and intensity is partially distributed into both sidebands for every $\Delta \varphi$. In contrast, a central feature of the $75-\mathrm{keV}$ data is a phase shift of $\sim 180^{\circ}$; each sideband maximizes at points where the other sideband intensity reaches zero.

Such an exclusive energy gain or loss as a function of laser delay implies that all the time-dependent electron density falls either purely on the accelerating or purely the decelerating slope of the laser acceleration cycles of the second interaction. Therefore, the time-modulated electron wave packet must consist of pulses at $\sim 100 \%$ temporal contrast, and there cannot be a substantial time-independent background current like so far observed in previous reports of laser-compressed electron pulses [8-10]. Figure 2(f) depicts the calculated temporal electron density at the revival conditions (see Appendix B). The background-free electron pulses (right panel) are slightly longer than for optimum compression (left panel), but they still have a full width at half maximum of approximately half the laser modulation period. Attosecond electron microscopy and diffraction [9] of stroboscopically excited material responses [10] are therefore advanced to the best possible imaging contrast if choosing $L=L_{\mathrm{QR}} / 2$ as the sample distance. Figure 2(g) shows the fit of the data in Fig. 2(d) to our analytical model. Almost all features are reproduced, and the measured states are therefore approximately pure states.

Taken together, the experimental and analytical results of Figs. 1 and 2 and the match of data with theory establish and affirm that laser-modulated free-electron wave packets with energy sidebands indeed reshape during free-space propagation through a series of fractional revivals back into the original wave function in the way depicted in Fig. 1(a). At half of the temporal Talbot distance, there is an ultrafast pulse train at $\sim 100 \%$ contrast.

\section{EVIDENCE FOR FREE-ELECTRON QUBITS}

The reported ability to interact multiple times in a coherent and time-controlled way with a free-electron wave function at distances of tens of centimeters creates a versatile perspective for more complex operations. We now report the formation of a free-electron qubit. The basis of our experiment is a recent theoretical proposal by Reinhardt et al. [22] on the use of laser-induced energy sidebands as a carrier of quantum information in a scheme where laser modulations and free-space propagations implement the noncommutative spin algebra of a qubit with all the necessary features [22]. However, we associate the qubit states $|0\rangle$ and $|1\rangle$ not with even and odd photon orders but directly with the -1 and +1 sidebands at $-h v$ and $+h v$, respectively. Figure 3 depicts the corresponding Bloch sphere. We assume that the laser-electron coupling strengths at the two interactions are equal and low enough to avoid higher-order sidebands and the onset of Rabi oscillations, that is, $\left|g_{1}\right|=\left|g_{2}\right|=|g|<\frac{1}{2}$. In this case, the superposition state $\alpha|0\rangle+\beta|1\rangle$ obtains at the fractional revival condition $L=L_{\mathrm{QR}} / 2$ the complex amplitudes:

$$
\begin{gathered}
\alpha=A \cos \left(\frac{\Delta \varphi}{2}-\frac{\pi}{4}\right), \quad \text { and } \\
\beta=A \exp \left(i \varphi_{0}\right) \sin \left(\frac{\Delta \varphi}{2}-\frac{\pi}{4}\right),
\end{gathered}
$$

where $A \propto J_{0}(|g|) J_{1}(|g|)$ depends on the laser power, $J_{0}$ and $J_{1}$ are the Bessel functions of the first kind, $\Delta \varphi=\arg g_{2}-$ 
$\arg g_{1}$ is the interferometric laser delay [see Fig. 2(a)], and $\varphi_{0}=\arg g_{1}+\arg g_{2}$ is the electron injection phase, that is, the absolute timing of the electron wave function with respect to the laser cycles. The values of $\alpha$ and $\beta$ have all the necessary properties of qubit amplitudes: They represent a closed system with $|\alpha|^{2}+|\beta|^{2}=$ const and can be set by the two laser-controlled parameters $\Delta \varphi$ and $\varphi_{0}$ to any point at the Bloch sphere [see Fig. 3(a)]. The first laser interaction (red arrow pointing left) creates a symmetric sideband pattern with $|\alpha|=|\beta|$. Free-space propagation (blue arrow) by $L_{\mathrm{QR}} / 2$ rotates the sideband phases [see Fig. 1(b)] and at the same time compresses the electron density to high-contrast pulses in the temporal domain [see Fig. 1(c)]. At this fractional revival, the second laser interaction (green arrows) creates the qubit in the above definition and simultaneously rotates it continuously around the Bloch sphere meridian in dependence on $\Delta \varphi$, the relative optical phase between the two laser waves in the experiment.

Figure 3(b) shows the measurement results. Plotted are the sideband spectra for $\Delta \varphi=\frac{1}{2} \pi, \frac{3}{2} \pi, \pi$, and 0 . We see the generation of the quantum states $|0\rangle,|1\rangle, \frac{1}{\sqrt{2}}(|0\rangle+|1\rangle)$, and $\frac{1}{\sqrt{2}}(|0\rangle-|1\rangle)$ in the form of a predominant production of the +1 sideband (first panel), a predominant production of the -1 sideband (second panel), and production of the two superposition states (third and fourth panel). Their phase differences are evident from Fig. 2(e) and can be determined with quantum state tomography [8]. Some residuals $(\sim 0.25)$ in the undesired sidebands for $|0\rangle$ and $|1\rangle$ are attributed to the remains of timing jitter in our laser-interferometric setup and residual mismatch of $L$ to $L_{\mathrm{QR}} / 2$. Nevertheless, the four states depicted on the Bloch sphere of Fig. 3(a) are resolved.

Given the above considerations, the reported results represent experimental evidence for the production of a qubit from a free electron as an elementary particle without an environment. The crucial steps are the laser-optical control of discrete electron energy sidebands and the discovery of fractional temporal revivals of such wave packets after free-space propagation. The measured transition from $|0\rangle$ to $\frac{1}{\sqrt{2}}(|0\rangle+|1\rangle)$ corresponds to applying an Hadamard operator [22], one of the two essential operations for implementing single-qubit algebra. Continuous phase rotation can be implemented by a further free-space propagation [blue dashed arrow in Fig. 3(a)] or by phase-locked electron injection via $\varphi_{0}$. Such a phase lock is achievable by laser-triggering the original electron emission timing or by preshaping the electron beam into pulses, for example, by time-locked bunching with microwave radiation [40] or terahertz fields [7]. In future experiments, coherent multicolor laser waves [8], terahertzdriven electron energy modulators [7], or discrete space-time coupling $[19,41]$ can expand the complexity of the laserdriven modulations, and electrostatic drift tubes or magnetic chicanes can set and reconfigure free-space propagation times at the frequency of electronics.

\section{DISCUSSION AND OUTLOOK}

The common mechanism behind the reported generation of maximum-contrast electron pulses and the formation of a free-electron qubit is the temporal Talbot effect and the ability of the free electron to interact multiple times in a coherent and time-controlled way with laser light at distances of tens of centimeters or more. In attosecond microscopy [42], the specimen can be placed at a far greater and more practical distance from the original temporal focus [43], that is, for optimum contrast at the half revivals [see Fig. 1(c)] or for optimum time resolution at any of the recurrences of the shortest pulses [see Fig. 1(d)]. Additionally, the fractional revivals provide means to generate more complicated temporal distributions of electrons for advanced time-imaging applications. If ultimately short pulses and high contrast are needed at the same time, two laser interactions can be combined [25]. For the qubits, there are probably alternative ways of moving around the Bloch sphere [22], for example, with the help of multifrequency lasers or special fractional revivals, to provide more flexible control than in our proof-of-principle demonstration.

By refraining from using femtosecond lasers and pulsed electron sources with their limited average brightness and instead modulating a continuous electron beam with continuous-wave lasers [10], the reported shaped electron wave packets in the form of ultrashort pulse trains or qubits are generated at almost the full brightness of the electron source, that is, at $\sim 10^{9}$ electrons/s in a nanometer beam, limited only by the current and emittance that can be delivered by modern high-energy electron sources. Transmission electron microscopes under the reported large-distance laser control may therefore become a versatile basis for novel types of fundamental and applied research with the free electron as an elementary particle.

\section{ACKNOWLEDGMENTS}

We acknowledge funding from the Dr. K. H. Eberle Stiftung, the Vector Stiftung, the European Research Council (ERC) via Consolidator Grant No. 647771, and the Deutsche Forschungsgemeinschaft (DFG) via SFB1432. We thank Johannes Thurner for help with the energy spectrometer, Guido Burkard for comments on the manuscript, and Ferenc Krausz for laboratory infrastructure.

\section{APPENDIX A: ANALYTICAL THEORY}

For the description of electron propagation and electronlight interaction, we apply a one-dimensional (1D) model with a fully coherent, pure electron wave function and classical light. We use the relativistically modified 1D Schrödinger equation $[27,44] i \hbar \partial_{t} \Psi(z, t)=\hat{H} \Psi(z, t)$ that accounts for the relativistic character of motion while maintaining the analytical and numerical simplicity of the Schrödinger equation. The electromagnetic fields around the membranes are calculated by multipath interference (see below). Electrons move in the $z$ direction with a velocity $v_{0}$ and experience two interactions with laser light. The central momentum is $p_{0}=$ $\gamma m_{e} v_{0}$, and the energy is $E_{0}=\gamma m_{e} c^{2}$, where $m_{e}$ is the rest mass of the electron, $\gamma=\left[1-\left(v_{0} / c\right)\right]^{-1 / 2}$ is the Lorentz factor, and $c$ is the speed of light. The values of $E_{0}$ and $p_{0}$ are weakly altered by the interaction which is therefore considered perturbative. Accordingly, the wave function is represented as a product of fast and slow parts [27], $\Psi(z, t)=$ $\psi\left(z^{\prime}, t\right) \exp \left[i\left(p_{0} z-E_{0} t\right) / \hbar\right]$, where $z^{\prime}=z-z$ is the local co- 
ordinate around the center of the wave packet $z_{0}=v_{0} t$. For free propagation, we take $\hat{H}=\hat{H}_{0}$, where $\hat{H}_{0}$ is the nonperturbed Hamiltonian, which is obtained by expansion of the electron energy $E$ around $E_{0}$ according to $\hat{H}_{0}=E_{0}+$ $\left(\hat{p}-p_{0}\right) v_{0}+\left(\hat{p}-p_{0}\right)^{2} /\left(2 \gamma^{3} m_{\mathrm{e}}\right)$, where $\hat{p}$ is the momentum operator [27]. Consequently, for free propagation, $\psi\left(z^{\prime}, t\right)$ satisfies

$$
i \hbar \partial_{t} \psi\left(z^{\prime}, t\right)=-\frac{\hbar^{2}}{2 m_{e} \gamma^{3}} \partial_{z^{\prime}}^{2} \psi\left(z^{\prime}, t\right) .
$$

The factor $m_{e} \gamma^{3}$ is sometimes called the longitudinal mass [45]. A similar equation for the envelope of the electron wave packet can be obtained by solving Dirac's equation [45]. In the following, we assume a Fourier-limited Gaussian wave packet with an energy spread of $\sigma_{E}$ and a length $\sigma_{z}=\hbar v_{0} /\left(2 \sigma_{E}\right)$ $[27,44]$ :

$$
\psi\left(z^{\prime}, 0\right)=\psi_{0} \exp \left(-\frac{z^{\prime 2}}{4 \sigma_{z}^{2}}\right),
$$

where $\psi_{0}=\left(2 \pi \sigma_{z}^{2}\right)^{-1 / 4}$ is the normalization coefficient. The temporal coherence is thereby considered a limiting factor for coherent interaction. After propagation from $z=0$ to $L_{\mathrm{D}}$, where $L_{\mathrm{D}}=v_{0} t_{\mathrm{D}}$ is the drift distance, and $t_{\mathrm{D}}$ is the drift time, the new wave function $\psi_{\mathrm{D}}\left(z^{\prime}\right)=\psi\left(z^{\prime}, t_{\mathrm{D}}\right)$ is

$$
\psi_{\mathrm{D}}\left(z^{\prime}\right)=\psi_{0} a_{\mathrm{D}}^{-1 / 2} \exp \left(-\frac{z^{\prime 2}}{4 \sigma_{z}^{2} a_{\mathrm{D}}}\right)
$$

where $a_{\mathrm{D}}=\left(1+i \xi L_{\mathrm{D}}\right)$ with dispersion parameter $\xi=$ $\hbar /\left(2 m_{e} \gamma^{3} \sigma_{z}^{2} v_{0}\right)=2 \sigma_{E}^{2} /\left(\hbar m_{e} \gamma^{3} v_{0}^{3}\right)$.

We now assume that the first interaction with the laser is centered at $z=L_{\mathrm{D}}$. For the interaction with the field, we use $\hat{H}=\hat{H}_{0}+\hat{H}_{\mathrm{I}}$, where the interaction part of the Hamiltonian is approximated as $\hat{H}_{\mathrm{I}} \approx-q_{e} A_{z} \hat{p} /\left(\gamma m_{e}\right), A_{z}$ is the $z$ component of the vector potential $[15,27,46]$, and $q_{e}$ is the negative-valued electron change. Here, we assume laser fields below the relativistic regime $\left|A_{z}\right| \ll\left|m_{e} c / q_{e}\right|$. With this modification, the equation for the interaction becomes

$$
i \hbar \partial_{t} \psi\left(z^{\prime}, t\right)=\left[-\frac{\hbar^{2}}{2 m_{e} \gamma^{3}} \partial_{z^{\prime}}^{2}+\frac{i \hbar q_{e}}{\gamma m_{e}} A_{z}\left(z^{\prime}+v_{0} t\right) \partial_{z^{\prime}}-\frac{q_{e} p_{0}}{\gamma m_{e}} A_{z}\left(z^{\prime}+v_{0} t\right)\right] \psi\left(z^{\prime}, t\right) .
$$

We now assume that the laser fields fade with distance from the interaction and vanish at $\left|z-L_{\mathrm{D}}\right|>v_{0} \Delta t_{1} / 2$, where the time scale of the interaction $\Delta t_{1}$ is small enough $\left(\Delta t_{1} \ll 1 / \xi\right)$ to neglect the envelope dispersion during the interaction, that is, the spatial derivatives in Eq. (A4). Solving the remaining first-order ordinary differential equation, we obtain

$$
\left.\psi\left(z^{\prime}, t_{\mathrm{D}}+\frac{\Delta \mathrm{t}_{1}}{2}\right)\right|_{\Delta t_{1} \rightarrow 0}=\left.\psi\left(z^{\prime}, t_{\mathrm{D}}-\frac{\Delta \mathrm{t}_{1}}{2}\right)\right|_{\Delta t_{1} \rightarrow 0} \exp \left[\frac{i q_{\mathrm{e}} v_{0}}{\hbar} \int d t^{\prime} A_{z}\left(z^{\prime}+v_{0} t^{\prime}, t^{\prime}\right)\right],
$$

where the integration is performed over an infinite interval; see also Refs. [27,45]. An interesting feature of Eq. (A5), also noted in Ref. [45], is that it is identical to its nonrelativistic counterpart, as considered in Refs. [11,15]. By using the complex electric field $\tilde{E}_{z}(z, t)=\tilde{E}_{z}(z) e^{-i \omega t}$, where $\omega$ is the laser central angular frequency, the vector potential is $A_{z}(z, t)=\left[\tilde{E}_{z}(z, t)-\tilde{E}_{z}^{*}(z, t)\right] /(2 \omega i)$. Here, we have assumed a continuous-wave laser, but the conclusions are also valid for laser pulses if their bandwidth is small. In the integrand of Eq. (A5), $\tilde{E}_{z}\left(z, t^{\prime}\right)=\tilde{E}_{z}(z) \exp \left(-i \omega z / v_{0}+i \omega z^{\prime} / v_{0}\right)$ and eventually

$$
\frac{q_{e} v_{0}}{\hbar} \int d t^{\prime} A_{z}\left(z^{\prime}+v_{0} t^{\prime}, t^{\prime}\right) \stackrel{t^{\prime}=\frac{z-z^{\prime}}{v_{0}}}{=} \frac{q_{e}}{\hbar \omega} \operatorname{Im}\left[\exp \left(\frac{i \omega z^{\prime}}{v_{0}}\right) \int d z \tilde{E}_{z}(z) \exp \left(-\frac{i \omega z}{v_{0}}\right)\right]=|g| \sin \left(k_{p} z^{\prime}+\varphi\right),
$$

where $\varphi=\arg g$, the modulation wave number $k_{p}=\omega / v_{0}$, and like in Refs. [11,27,45],

$$
g=\frac{q_{e}}{\hbar \omega} \int d z \tilde{E}_{z}(z) \exp \left(-\frac{i \omega z}{v_{0}}\right)
$$

is the coupling strength, sometimes also called the PINEM field $[11,17,18]$.

\section{Laser fields and coupling strengths}

For our interaction setting, a semiconductor slab of refractive index $n_{s}$ and thickness $d_{s}$ illuminated by a laser beam such that the focus size is large compared to the wavelength, the laser fields are approximated as plane waves with the amplitudes adiabatically decaying to infinity, and

$$
\begin{aligned}
g= & g\left(E_{0}, \omega, \alpha, \alpha_{e}, v_{0}, n_{s}, d_{s}\right)=\frac{q_{\mathrm{e}} v_{0} E_{0} \exp (i \phi)}{i \hbar \omega^{2}}\left\{\frac{\sin \left(\alpha-\alpha_{e}\right)}{1-\beta \cos \left(\alpha-\alpha_{e}\right)}+r \frac{\sin \left(\alpha+\alpha_{e}\right)}{1+\beta \cos \left(\alpha+\alpha_{e}\right)}\right. \\
& \left.+t_{\text {slab }}\left[\frac{\sin \left(\alpha_{2}-\alpha_{e}\right)\left(e^{-i \kappa}-1\right)}{1-n_{s} \beta \cos \left(\alpha_{2}-\alpha_{e}\right)}+r_{\text {slab }} \frac{\sin \left(\alpha_{2}+\alpha_{e}\right)\left(e^{-i \eta}-1\right)}{1+n_{s} \beta \cos \left(\alpha_{2}+\alpha_{e}\right)}\right]-t \frac{\sin \left(\alpha-\alpha_{e}\right)}{1-\beta \cos \left(\alpha-\alpha_{e}\right)} e^{-i \zeta}\right\},
\end{aligned}
$$

where $E_{0}$ is the amplitude, and $\phi$ is the phase of the $z$ component of the electric field at the first interface of the membrane, $\alpha$ and $\alpha_{e}$ are, respectively, the angles of incidence of the laser and the electron beam, $\alpha_{2}=\operatorname{asin}\left(\sin \alpha / n_{s}\right)$ is the refraction 
angle, $\quad \kappa=\omega\left[1-n_{s} \beta \cos \left(\alpha_{2}-\alpha_{e}\right)\right] /\left(v_{0} \cos \alpha_{e}\right), \quad \eta=$ $\omega\left[1+n_{s} \beta \cos \left(\alpha_{2}+\alpha_{e}\right)\right] /\left(v_{0} \cos \alpha_{e}\right), \quad \zeta=\omega[1-\beta \cos (\alpha-$ $\left.\left.\alpha_{e}\right)-\frac{\omega}{c} d \cos \alpha\right] /\left(v_{0} \cos \alpha_{e}\right), r=r_{12}\left(1-e^{2 i \delta}\right) /\left(1-r_{12}^{2} e^{2 i \delta}\right)$ is the reflection coefficient for the slab, $t_{\text {slab }}=t_{12} /\left(1-r_{12}^{2} e^{2 i \delta}\right)$ and $r_{\text {slab }}=-r_{12} e^{i 2 \delta}$ describe the fields within the slab, $t=\left(1-r_{12}^{2}\right) e^{i \delta} /\left(1-r_{12}^{2} e^{2 i \delta}\right)$ is the transmission coefficient, $\delta=\frac{\omega}{c} n_{s} d_{s} \cos \alpha_{2}$, and $r_{12}$ and $t_{12}$ are the Fresnel reflection and transmission coefficients for $p$-polarization at a boundary of vacuum and the membrane material, respectively.

\section{Modulation, propagation, and revivals}

Using Eqs. (A5)-(A7), we obtain the wave function $\psi_{1}\left(z^{\prime}\right)=\left.\psi\left(z^{\prime}, t_{\mathrm{D}}+\Delta t_{1} / 2\right)\right|_{\Delta t_{1} \rightarrow 0}$ after the first interaction:

$$
\psi_{1}\left(z^{\prime}\right)=\psi_{\mathrm{D}}\left(z^{\prime}\right) \exp \left[i\left|g_{1}\right| \sin \left(k_{p} z^{\prime}+\varphi_{1}\right)\right]
$$

where the indices 1 indicate parameters related to the first interaction. A similar equation for the envelope of the electron wave packet can alternatively be obtained by solving Dirac's equation [45]. We decompose the exponential in Eq. (A9) by using the Jacobi-Anger expansion [26] $\exp \left[i\left|g_{1}\right| \sin \left(k_{p} z^{\prime}+\varphi_{1}\right)\right]=\sum_{n} J_{n}\left(\left|g_{1}\right|\right) \exp \left(i n k_{p} z^{\prime}+i n \varphi_{1}\right)$, where $J_{n}$ is the Bessel function of the first kind, and the summation is hereon performed over the integer indices from $-\infty$ to $+\infty$. By applying a Fourier transform $\tilde{\psi}_{1}(k)=\int_{-\infty}^{\infty} \psi_{1}\left(z^{\prime}\right) e^{-i k z^{\prime}} d z^{\prime}$ of the resulting equation, where $k$ is the Fourier conjugate to the coordinate $z^{\prime}$, and replacing $E^{\prime}=\hbar k v_{0}, \hbar \omega=\hbar k_{p} v_{0}$, where $E^{\prime}=E-E_{0}$ is the offset energy of the electron, we obtain the spectrum in energy units:

$$
\tilde{\psi}_{1}\left(E^{\prime}\right)=\tilde{\psi}_{0} \sum_{n} J_{n}\left(\left|g_{1}\right|\right) e^{i n \varphi_{1}} \exp \left[-\frac{\left(E^{\prime}-n \hbar \omega\right)^{2} a_{\mathrm{D}}}{4 \sigma_{E}^{2}}\right],
$$

where $\tilde{\psi}_{0}=\psi_{0} \sqrt{4 \pi \sigma_{z}^{2}}$. Variables with tildes denote spectral quantities. The further evolution of the wave function is again obtained by solving Eq. (A1) in the spectral domain. At a distance $L$ after the first interaction, the propagated wave function in the momentum space is

$$
\begin{aligned}
\tilde{\psi}_{L}\left(E^{\prime}\right) & =\tilde{\psi}_{1}\left(E^{\prime}\right) \exp \left(-\frac{i E^{\prime 2} L}{2 m_{e} \gamma^{3} \hbar v_{0}^{2}}\right) \\
& =\tilde{\psi}_{1}\left(E^{\prime}\right) \exp \left[-i \pi \frac{E^{\prime 2}}{(h v)^{2}} \frac{L}{L_{\mathrm{QR}}}\right],
\end{aligned}
$$

where the quantum-revival distance $L_{\mathrm{QR}}=\beta^{3} \gamma^{3} \lambda^{2} m_{e} c / h$ is introduced; in the coordinate space, the propagated wave function is

$$
\begin{aligned}
\psi_{L}\left(z^{\prime}\right)= & \psi\left(z^{\prime}, 0\right) \sum_{n} J_{n}\left(\left|g_{1}\right|\right) \exp \left(\frac{i \omega n z^{\prime}}{v_{0}}+i n \varphi_{1}\right) \\
& \times \exp \left(-i \pi n^{2} \frac{L}{L_{\mathrm{QR}}} \frac{a_{\mathrm{D}}}{a_{\mathrm{L}}}\right),
\end{aligned}
$$

where $a_{L}=1+i \xi\left(L_{\mathrm{D}}+L\right)$. If the temporal coherence is large $\left(\xi L_{\mathrm{D}} \ll 1\right.$ and $\left.\xi L \ll 1\right)$, the dispersion of the envelope in Eq. (A12) can be neglected. By setting $a_{\mathrm{D}}=a_{L}=1$, we arrive at

$$
\begin{aligned}
\psi_{L}\left(z^{\prime}\right)= & \psi\left(z^{\prime}, 0\right) \sum_{n} J_{n}\left(\left|g_{1}\right|\right) \exp \left(\frac{i \omega n z^{\prime}}{v_{0}}+i n \varphi_{1}\right) \\
& \times \exp \left(-i \pi n^{2} \frac{L}{L_{\mathrm{QR}}}\right) .
\end{aligned}
$$

Separating into odd and even sidebands, we obtain from Eq. (A13)

$$
\begin{aligned}
\psi_{L}\left(z^{\prime}\right)= & \psi_{0} \exp \left(-\frac{z^{\prime 2}}{4 \sigma_{z}^{2}}\right)\left[J_{0}\left(\left|g_{1}\right|\right)+2 i \sum_{n=1,3, \ldots} J_{n}\left(\left|g_{1}\right|\right)\right. \\
& \times \sin \left(\frac{\omega n z^{\prime}}{v_{0}}\right) \exp \left(-i \pi n^{2} \frac{L}{L_{\mathrm{QR}}}\right) \\
& \left.+2 \sum_{n=2,4, \ldots} J_{n}\left(\left|g_{1}\right|\right) \cos \left(\frac{\omega n z^{\prime}}{v_{0}}\right) \exp \left(-i \pi n^{2} \frac{L}{L_{\mathrm{QR}}}\right)\right] .
\end{aligned}
$$

In Eq. (A14), one can explicitly see the quadratic dependencies of sideband phases on their number, the restoration of phases at $L=L_{\mathrm{QR}}$, and the $\pi / 2$ phase shift between the odd and even sidebands in the initial wave function $(L=0)$ that is compensated under the half-revival conditions $\left(L=L_{\mathrm{QR}} / 2\right)$, producing $100 \%$ temporal contrast [see Fig. 1(b)].

Equation (A12) allows us to estimate the conditions for observation of the revivals for a typical transmission electron microscope at $E_{0}=75 \mathrm{keV}$ with a $0.2 \mathrm{eV}$ full width at half maximum energy spread $\left(\sigma_{E}=0.085 \mathrm{eV}\right)$; we obtain $\xi \approx 0.8 \mathrm{~m}^{-1}$. Therefore, the energy spread of a typical microscope without monochromator is sufficient to see revivals after tens of centimeters of propagation. Distances at which revivals can be observed increase quadratically with the inverse bandwidth. A microscope with an energy monochromator therefore promises several meters of dispersion-free revivals.

\section{Second laser-electron interaction}

If a second laser-electron interaction is located at a distance $L$ after the first stage, the laser imposes a second phase modulation on the wave function in analogy to Eq. (A13):

$$
\psi_{2}\left(z^{\prime}\right)=\psi_{L}\left(z^{\prime}\right) \sum_{m} J_{m}\left(\left|g_{2}\right|\right) \exp \left(i m k_{p} z^{\prime}+i m \varphi_{2}\right),
$$

where $g_{2}$ and $\varphi_{2}$ are the coupling constant and its phase for the second interaction, respectively. By applying the inverse Fourier transform, separating the real and imaginary parts in the phase terms and introducing the photon order $N=n+m$, we obtain from Eq. (A15) the spectrum after the second interaction:

$$
\begin{aligned}
& \tilde{\psi}_{2}\left(E^{\prime}, \Delta \varphi\right) \\
& =\sum_{N, n} \tilde{\psi}_{N} J_{n}\left(\left|g_{1}\right|\right) J_{N-n}\left(\left|g_{2}\right|\right) \exp [i(N-n) \Delta \varphi] \\
& \quad \times \exp \left(-i 2 \pi \frac{L}{L_{\mathrm{QR}}} n \frac{E^{\prime}-\hbar \omega N}{\hbar \omega}\right) \exp \left(-i \pi n^{2} \frac{L}{L_{\mathrm{QR}}}\right),
\end{aligned}
$$


where $\tilde{\psi}_{N}=\tilde{\psi}_{0} \exp \left(i N \varphi_{1}\right) \exp \left[-a_{\mathrm{L}}\left(E^{\prime}-N \hbar \omega\right)^{2} / 4 \sigma_{E}^{2}\right]$. Equation (A16) represents a set of peaks at photon orders $N$, centered at energies $N \hbar \omega$. Each of the peaks is composed of several components originating from virtual absorption of $n$ and $N-n$ photons on the first and second stages, respectively [17]. From Eq. (A16), we see that, at the exact photon orders $\left|E^{\prime}-\hbar \omega N\right| \ll \hbar \omega$, the phases of the sidebands acquire particular values at distances equal to the multiples of $L_{\mathrm{QR}} / 2$; see also Ref. [22].

\section{Qubit amplitudes}

If we assume $\left|g_{1}\right|=\left|g_{2}\right|=|g|<\frac{1}{2}$ for Eq. (A16), we can neglect all sidebands except for $n=0, \pm 1$ and arrive at expressions for the \pm 1 sidebands in the spectral domain at the exact value of the photon orders $\left|E^{\prime}-\hbar \omega N\right| \ll \hbar \omega$ :

$$
\begin{aligned}
\tilde{\psi}_{2}( \pm h v, \Delta \varphi)= & \pm 2\left|\tilde{\psi}_{0}\right|^{2} J_{0}(|g|) J_{1}(|g|) \\
& \times \exp \left[i \frac{\frac{\pi L}{L_{\mathrm{QR}}} \pm\left(\varphi_{1}+\varphi_{2}\right)}{2}\right] \cos \left(\frac{\frac{\pi L}{L_{\mathrm{QR}}} \pm \Delta \varphi}{2}\right) .
\end{aligned}
$$

By removing the common phase, we obtain

$$
\tilde{\psi}_{2}(-h v, \Delta \varphi)=2\left|\tilde{\psi}_{0}\right|^{2} J_{0}(|g|) J_{1}(|g|) \cos \left(\frac{\frac{\pi L}{L_{\mathrm{R}}}-\Delta \varphi}{2}\right), \quad \text { and }
$$

$$
\begin{aligned}
\tilde{\psi}_{2}(+h v, \Delta \varphi)= & -2\left|\tilde{\psi}_{0}\right|^{2} J_{0}(|g|) J_{1}(|g|) \exp \left[i\left(\varphi_{1}+\varphi_{2}\right)\right] \\
& \times \cos \left(\frac{\frac{\pi L}{L_{\mathrm{QR}}}+\Delta \varphi}{2}\right) .
\end{aligned}
$$

At the fractional revival condition $L / L_{\mathrm{QR}}=\frac{1}{2}$, we obtain Eqs. (1) and (2) for the qubit amplitudes $\alpha$ and $\beta$.

\section{Amount of the revivals in the time domain}

To understand the consequences of a limited coherence to the amount of the revivals that can in principle be observed, we consider some drift distance $L_{\mathrm{D}}$ of the initially minimal Gaussian wave packet before the first interaction. The wave function in the time domain is

$$
\begin{aligned}
\psi_{L}\left(z^{\prime}, t\right)= & \psi_{0} a_{L}^{-\frac{1}{2}} \sum_{n} J_{n}\left(\left|g_{1}\right|\right) \exp \left(i n \varphi_{1}\right) \\
& \times \exp \left[-\frac{\left(z^{\prime}-n \lambda \beta \frac{L}{L_{\mathrm{QR}}}\right)^{2}}{4 \sigma_{z}^{2}\left|a_{L}\right|^{2}}\right] \\
& \times \exp \left\{i \frac { 1 } { | a _ { L } | ^ { 2 } } \left[\left(n k_{p} z^{\prime}-n^{2} \pi \frac{L}{L_{\mathrm{QR}}}\right)\right.\right. \\
& \left.\left.\times \operatorname{Re}\left(a_{\mathrm{D}} a_{L}^{*}\right)+\frac{\operatorname{Im}\left(a_{L}\right)}{4 \sigma_{z}^{2}} z^{\prime 2}\right]\right\},
\end{aligned}
$$

where the factors $a_{\mathrm{D}}=1+i \xi L_{\mathrm{D}}$ and $a_{L}=1+i \xi\left(L_{\mathrm{D}}+L\right)$ account for the dispersion before and after the interaction, and the dispersion parameter $\xi=\hbar /\left(2 m_{e} \gamma^{3} \sigma_{z}^{2} v_{0}\right)=$ $2 \sigma_{E}^{2} /\left(m_{e} \gamma^{3} v_{0}^{3} \hbar\right)$. By analyzing the phases of the components in Eq. (A20), we obtain the limiting number of revivals that are permitted for a sideband $n$ by the envelope spread:

$$
\ell_{\lim }=\frac{\pi}{2} N_{\text {cycles }}^{2} n^{2},
$$

where $N_{\text {cycles }}$ is the length of the wave packet at the first interaction stage divided by the modulation period; $N_{\text {cycles }}=$ $2 \sigma_{z} \sqrt{1+\xi^{2} L_{\mathrm{D}}^{2}} /(\lambda \beta)$. To obtain the condition for revival of all sidebands from Eq. (A21), one should substitute $n=1$. Interestingly, the pre-interaction drift $L_{\mathrm{D}}$ alleviates the condition of Eq. (A21) by spreading the wave packet out over several cycles or light.

Also, group dispersion of the wave packet envelopes can prevent the revivals in the time domain, when the slippage of the envelopes is larger than the envelope itself. In this case, the amount of the revivals is limited by the mutual slippage of the envelopes of the sidebands and equals as follows from Eq. (A20)

$$
\ell_{\max } \approx \frac{N_{\text {cycles }}}{n_{\max }}=\frac{2 \sigma_{z}}{n_{\max } \lambda \beta} \approx \frac{\hbar \omega}{4 \pi \sigma_{E}|g|},
$$

where we have estimated the maximum number of the nonnegligible sideband as $n_{\max } \approx 2|g|$. In the spectral domain, the slippage of the envelopes to more than the width of the envelope will produce interference fringes inside each sideband.

\section{APPENDIX B: FIGURE DETAILS}

The temporal electron density in Fig. 1(d) is calculated with Eq. (A12) for a propagation distance of $L=12 \mathrm{~cm}$, a pre-interaction drift distance $L_{\mathrm{D}}=0$, a coupling strength of $|g|=0.785$, and a bandwidth $\sigma_{E}=0.03 \mathrm{eV}$. The pulse duration $\Delta \tau$ in Fig. 1(e) is calculated as the full width at half maximum of the central maximum of electron density above the adjacent minima. The temporal contrast in Fig. 1(e) is calculated as the ratio of the difference of the central density maximum and its adjacent minimum to their sum.

To determine the effective coupling factors $\left|g_{1,2}\right|$ of the experiment, we record electron spectra from each laser-electron interaction alone and fit the resulting data with $\left|\tilde{\psi}_{1}\left(E^{\prime}\right)\right|^{2}$, see Eq. (A10); $|g|$ is the only parameter. We obtain $\left|g_{1}\right| \approx$ $\left|g_{2}\right| \approx 0.5$ and 0.3 for 120 and $75 \mathrm{keV}$, respectively. These values approximately match the expectation from Eq. (A8). Using these coupling constants, the delay-dependent data of Fig. 2(d) is fitted with $\left|\tilde{\psi}_{2}\left(E^{\prime}, \Delta \varphi\right)\right|^{2}$, see Eq. (A16). Two fit parameters are applied: an experimental offset to $\Delta \varphi$ and the initial electron energy spread $\sigma_{\text {exp }}$. Figure $2(\mathrm{~g})$ shows a comparison of the result and the experimental data of Fig. 2(d). The fit reproduces the positions of all peaks and minima, their widths and mutual shifts, as well as their amplitudes. These results confirm that almost all electrons in the beam are modulated into attosecond pulses and qubits in accordance with our report.

The amplitudes of the sidebands in Fig. 2(e) are measured by fitting the measured data with three peaks at 0 and $\pm h v$ followed by convolution with the laser-unaffected zero-loss peak. The temporal electron density in Fig. 2(f) is calculated as an incoherent superposition of wave packets with random emission timing for a propagation distance of $12 \mathrm{~cm}$, a coupling strength of $|g|=0.785$, and a coherence length 
corresponding to a bandwidth of $0.2 \mathrm{eV}$ (full width at half maximum) or $\sigma_{E}=0.085 \mathrm{eV}$.

\section{APPENDIX C: RELATIONS TO QUANTUM REVIVALS IN OTHER FIELDS}

Our analytical equation for the temporal revival distance $L_{\mathrm{QR}}=d^{2} / \lambda_{e}$ has a very similar shape [23] to the equation for the optical Talbot distance $z_{\mathrm{T}}=a^{2} / \lambda$ for the diffraction of light with a wavelength $\lambda$ from a grating with period $a[28,29,47,48]$. In that respect, the measured revival phe- nomenon is called a temporal electronic Talbot effect. Related quantum revivals [49] have also been observed in a variety of quantum systems other than free electrons, for example, in isolated atoms and molecules [31-33], many-body quantum systems [34-38], and spin systems [39]. In optics, transverse revivals [50,51] are used for diffraction-free imaging [52,53], nano-assembly [54], optical tweezers [55], or x-ray imaging [56,57]. Transverse revivals of matter waves are used for interferometry and high-resolution lithography [58-61]. Our measurement results add matter-wave revivals in the temporal dimension to this group.
[1] A. Polman, M. Kociak, and F. J. García de Abajo, Electronbeam spectroscopy for nanophotonics, Nat. Mater. 18, 1158 (2019).

[2] O. Kfir, Entanglements of Electrons and Cavity Photons in the Strong-Coupling Regime, Phys. Rev. Lett. 123, 103602 (2019).

[3] O. Kfir, H. Lourenço-Martins, G. Storeck, M. Sivis, T. R. Harvey, T. J. Kippenberg, A. Feist, and C. Ropers, Controlling free electrons with optical whispering-gallery modes, Nature (London) 582, 46 (2020).

[4] A. Gover and A. Yariv, Free-Electron-Bound-Electron Resonant Interaction, Phys. Rev. Lett. 124, 064801 (2020).

[5] C. Mechel, Y. Kurman, A. Karnieli, N. Rivera, A. Arie, and I. Kaminer, Quantum correlations in electron microscopy, Optica 8, 70 (2021).

[6] A. Ben Hayun, O. Reinhardt, J. Nemirovsky, A. Karnieli, N. Rivera, and I. Kaminer, Shaping quantum photonic states using free electrons, Sci. Adv. 7, eabe4270 (2021).

[7] C. Kealhofer, W. Schneider, D. Ehberger, A. Ryabov, F. Krausz, and P. Baum, All-optical control and metrology of electron pulses, Science 352, 429 (2016).

[8] K. E. Priebe, C. Rathje, S. V. Yalunin, T. Hohage, A. Feist, S. Schäfer, and C. Ropers, Attosecond electron pulse trains and quantum state reconstruction in ultrafast transmission electron microscopy, Nat. Photonics 11, 793 (2017).

[9] Y. Morimoto and P. Baum, Diffraction and microscopy with attosecond electron pulse trains, Nat. Phys. 14, 252 (2018).

[10] A. Ryabov, J. W. Thurner, D. Nabben, M. V. Tsarev, and P. Baum, Attosecond metrology in a continuous-beam transmission electron microscope, Sci. Adv. 6, eabb1393 (2020).

[11] S. T. Park, M. Lin, and A. H. Zewail, Photon-induced near-field electron microscopy (PINEM): Theoretical and experimental, New J. Phys. 12, 123028 (2010).

[12] E. Pomarico, I. Madan, G. Berruto, G. M. Vanacore, K. Wang, I. Kaminer, J. F. García de Abajo, and F. Carbone, meV resolution in laser-assisted energy-filtered transmission electron microscopy, ACS Photonics 5, 759 (2018).

[13] A. Ryabov and P. Baum, Electron microscopy of electromagnetic waveforms, Science 353, 374 (2016).

[14] I. Madan, G. M. Vanacore, E. Pomarico, G. Berruto, R. J. Lamb, D. McGrouther, T. T. A. Lummen, T. Latychevskaia, F. J. García de Abajo, and F. Carbone, Holographic imaging of electromagnetic fields via electron-light quantum interference, Sci. Adv. 5, eaav8358 (2019).

[15] F. J. García de Abajo and M. Kociak, Electron energy-gain spectroscopy, New J. Phys. 10, 073035 (2008).
[16] B. Barwick, D. J. Flannigan, and A. H. Zewail, Photoninduced near-field electron microscopy, Nature (London) 462, 902 (2009).

[17] A. Feist, K. E. Echternkamp, J. Schauss, S. V. Yalunin, S. Schäfer, and C. Ropers, Quantum coherent optical phase modulation in an ultrafast transmission electron microscope, Nature (London) 521, 200 (2015).

[18] K. E. Echternkamp, A. Feist, S. Schäfer, and C. Ropers, Ramsey-type phase control of free-electron beams, Nat. Phys. 12, 1000 (2016).

[19] G. M. Vanacore, I. Madan, G. Berruto, K. Wang, E. Pomarico, R. J. Lamb, D. McGrouther, I. Kaminer, B. Barwick, F. J. García de Abajo, and F. Carbone, Attosecond coherent control of free-electron wave functions using semi-infinite light fields, Nat. Commun. 9, 2694 (2018).

[20] F. Hasselbach, Progress in electron- and ion-interferometry, Rep. Prog. Phys. 73, 016101 (2009).

[21] P. Baum and F. Krausz, Capturing atomic-scale carrier dynamics with electrons, Chem. Phys. Lett. 683, 57 (2017).

[22] O. Reinhardt, C. Mechel, M. Lynch, and I. Kaminer, Freeelectron qubits, Ann. Phys. Lpz. 533, 2000254 (2020).

[23] V. Di Giulio and F. J. García de Abajo, Free-electron shaping using quantum light, Optica 7, 1820 (2020).

[24] P. Baum and A. H. Zewail, Attosecond electron pulses for 4D diffraction and microscopy, Proc. Natl. Acad. Sci. USA 104, 18409 (2007).

[25] S. V. Yalunin, A. Feist, and C. Ropers, Tailored high-contrast attosecond electron pulses for coherent excitation and scattering, Phys. Rev. Research 3, L032036 (2021).

[26] M. Abramowitz and I. A. Stegun, Handbook of Mathematical Functions, with Formulas, Graphs, and Mathematical Tables (Dover, New York, 1965).

[27] A. Gover and Y. Pan, Dimension-dependent stimulated radiative interaction of a single electron quantum wavepacket, Phys. Lett. A 382, 1550 (2018).

[28] L. Rayleigh F. R. S., XXV. On copying diffraction-gratings, and on some phenomena connected therewith, Philos. Mag. 11, 196 (1881).

[29] S. Teng, N. Zhang, Q. Dong, and C. Cheng, Diffraction of a one-dimensional phase grating in the deep Fresnel field, J. Opt. Soc. Am. A 24, 3636 (2007).

[30] E. Essers, G. Benner, T. Mandler, S. Meyer, D. Mittmann, M. Schnell, and R. Hoschen, Energy resolution of an Omega-type monochromator and imaging properties of the MANDOLINE filter, Ultramicroscopy 110, 971 (2010). 
[31] G. Rempe, H. Walther, and N. Klein, Observation of Quantum Collapse and Revival in a One-Atom Maser, Phys. Rev. Lett. 58, 353 (1987).

[32] P. T. Greenland, S. A. Lynch, A. F. G. van der Meer, B. N. Murdin, C. R. Pidgeon, B. Redlich, N. Q. Vinh, and G. Aeppli, Coherent control of Rydberg states in silicon, Nature (London) 465, 1057 (2010).

[33] F. Vagizov, V. Antonov, Y. V. Radeonychev, R. N. Shakhmuratov, and O. Kocharovskaya, Coherent control of the waveforms of recoilless gamma-ray photons, Nature (London) 508, 80 (2014).

[34] M. Greiner, O. Mandel, T. W. Hansch, and I. Bloch, Collapse and revival of the matter wave field of a Bose-Einstein condensate, Nature (London) 419, 51 (2002).

[35] S. Will, T. Best, U. Schneider, L. Häckermuller, D. S. Luhmann, and I. Bloch, Time-resolved observation of coherent multi-body interactions in quantum phase revivals, Nature (London) $\mathbf{4 6 5}$, 197 (2010)

[36] B. Rauer, S. Erne, T. Schweigler, F. Cataldini, M. Tajik, and J. Schmiedmayer, Recurrences in an isolated quantum many-body system, Science 360, 307 (2018).

[37] P. Schlagheck, D. Ullmo, J. D. Urbina, K. Richter, and S. Tomsovic, Enhancement of Many-Body Quantum Interference in Chaotic Bosonic Systems: The Role of Symmetry and Dynamics, Phys. Rev. Lett. 123, 215302 (2019).

[38] P. T. Grochowski, T. Karpiuk, M. Brewczyk, and K. Rzazewski, Fermionic quantum carpets: From canals and ridges to solitonlike structures, Phys. Rev. Research 2, 013119 (2020).

[39] V. Kumar Vimal and V. Subrahmanyam, Magnetization revivals and dynamics of quantum correlations in a Kitaev spin chain, Phys. Rev. A 102, 012406 (2020).

[40] X. Fu, E. Wang, Y. Zhao, A. Liu, E. Montgomery, V. J. Gokhale, J. J. Gorman, C. Jing, J. W. Lau, and Y. Zhu, Direct visualization of electromagnetic wave dynamics by laser-free ultrafast electron microscopy, Sci. Adv. 6, eabc3456 (2020).

[41] A. Feist, S. V. Yalunin, S. Schäfer, and C. Ropers, High-purity free-electron momentum states prepared by three-dimensional optical phase modulation, Phys. Rev. Research 2, 043227 (2020).

[42] K. J. Mohler, D. Ehberger, I. Gronwald, C. Lange, R. Huber, and P. Baum, Ultrafast electron diffraction from nanophotonic waveforms via dynamical Aharonov-Bohm phases, Sci. Adv. 6, eabc8804 (2020).

[43] P. Baum, Quantum dynamics of attosecond electron pulse compression, J. Appl. Phys. 122, 223105 (2017).

[44] A. Friedman, A. Gover, G. Kurizki, S. Ruschin, and A. Yariv, Spontaneous and stimulated emission from quasifree electrons, Rev. Mod. Phys. 60, 471 (1988).

[45] S. T. Park and A. H. Zewail, Relativistic effects in photoninduced near field electron microscopy, J. Phys. Chem. A 116, 11128 (2012).
[46] J. F. García de Abajo, A. Asenjo-Garcia, and M. Kociak, Multiphoton absorption and emission by interaction of swift electrons with evanescent light fields, Nano Lett. 10, 1859 (2010).

[47] W. B. Case, M. Tomandl, S. Deachapunya, and M. Arndt, Realization of optical carpets in the Talbot and Talbot-Lau configurations, Opt. Express 17, 20966 (2009).

[48] D. Dragoman and M. Dragoman, Quantum-Classical Analogies (Springer-Verlag, Berlin Heidelberg, 2004).

[49] R. Robinett, Quantum wave packet revivals, Phys. Rep. 392, 1 (2004).

[50] J. Azaña and H. Guillet de Chatellus, Angular Talbot Effect, Phys. Rev. Lett. 112, 213902 (2014).

[51] Y. Lumer, L. Drori, Y. Hazan, and M. Segev, Accelerating SelfImaging: The Airy-Talbot effect, Phys. Rev. Lett. 115, 013901 (2015).

[52] H. Guillet de Chatellus, L. Romero Cortés, A. Deville, M. Seghilani, and J. Azaña, Diffraction-Induced Bidimensional Talbot Self-Imaging with Full Independent Period Control, Phys. Rev. Lett. 118, 133903 (2017).

[53] R.-E. Lu, R.-z. Zhao, X. Feng, B. Yang, X.-H. Hong, C. Zhang, Y.-Q. Qin, and Y.-Y. Zhu, Nearly Diffraction-Free Nonlinear Imaging of Irregularly Distributed Ferroelectric Domains, Phys. Rev. Lett. 120, 067601 (2018).

[54] D. Ohl de Mello, D. Schäffner, J. Werkmann, T. Preuschoff, L. Kohfahl, M. Schlosser, and G. Birkl, Defect-Free Assembly of 2D Clusters of More Than 100 Single-Atom Quantum Systems, Phys. Rev. Lett. 122, 203601 (2019).

[55] B. P. da Silva, V. A. Pinillos, D. S. Tasca, L. E. Oxman, and A. Z. Khoury, Pattern Revivals from Fractional Gouy Phases in Structured Light, Phys. Rev. Lett. 124, 033902 (2020).

[56] P. Cloetens, J. P. Guigay, C. D. Martino, J. Baruchel, and M. Schlenker, Fractional Talbot imaging of phase gratings with hard x rays, Opt. Lett. 22, 1059 (1997).

[57] M. Seifert, V. Ludwig, and S. Kaeppler, Talbot-Lau x-ray phasecontrast setup for fast scanning of large samples, Sci. Rep. 9 , 4199 (2019).

[58] L. Hackermuller, K. Hornberger, B. Brezger, A. Zeilinger, and M. Arndt, Decoherence of matter waves by thermal emission of radiation, Nature (London) 427, 711 (2004).

[59] S. Gerlich, L. Hackermüller, K. Hornberger, A. Stibor, H Ulbricht, M. Gring, F. Goldfarb, T. Savas, M. Müri, M. Mayor, and M. Arndt, A Kapitza-Dirac-Talbot-Lau interferometer for highly polarizable molecules, Nat. Phys. 3, 711 (2007).

[60] A. D. Cronin, J. Schmiedmayer, and D. E. Pritchard, Optics and interferometry with atoms and molecules, Rev. Mod. Phys. 81, 1051 (2009).

[61] B. J. McMorran and A. D. Cronin, An electron Talbot interferometer, New J. Phys. 11, 033021 (2009). 